\title{
SIMPLE NEAR-RINGS OF DIFFERENTIABLE TRANSFORMATIONS
}

\author{
D. W. BLACKETT
}

An earlier paper [ 1 ] reduced the study of semisimple near-rings to the study of simple near-rings. The definition of a radical for nearrings by W. E. Deskins [2] permits a partial description of many non-semisimple near-rings in terms of simple near-rings. The present paper explores the structure of some simple near-rings.

Represent vectors of an $n$-dimensional vector space $V$ over the real numbers by their coordinates

$X=\left(x_{1}, \cdots, x_{n}\right), \quad Y=\left(y_{1}, \cdots, y_{n}\right), \quad A=\left(a_{1}, \cdots, a_{n}\right), \cdots$

with respect to a fixed basis of $V$. A transformation $T: X \rightarrow X T=Y$ of $V$ into itself may be described by $n$ coordinate functions

$$
y_{i}=f^{i}\left(x_{1}, \cdots, x_{n}\right)=f^{i}(X)
$$

or by the $n$-tuple

$$
Y=X T=F(X)=\left(f^{1}(X), \cdots, f^{n}(X)\right) .
$$

The $i$ th partial derivative of $F(X)$ at $A$ is

$$
F_{i}(A)=\left(f_{i}^{1}(A), \cdots, f_{i}^{n}(A)\right)
$$

where $f_{i}^{j}(A)$ is the $i$ th partial derivative of $f^{i}(X)$ evaluated at $A$. The transformation $T$ is differentiable at $A$ if the $n$ partial derivatives of $F(X)$ exist in a neighborhood of $A$ and are continuous at $A$. The total differential at $A$ of a transformation $T$ which is differentiable at $A$ is

$$
\Delta F(A)=F_{1}(A) \Delta x_{1}+\cdots+F_{n}(A) \Delta x_{n} .
$$

THEOREM 1. Let $N$ be a near-ring (under addition and iteration) of transformations of $V$ which have origin $\theta$ of $V$ as a fixed point and also as a point of differentiability. The correspondence

$$
T \rightarrow\left(\begin{array}{ccc}
f_{1}^{1}(\theta) & \cdots & f_{1}^{n}(\theta) \\
\vdots & & \vdots \\
f_{n}^{1}(\theta) & \cdots & f_{n}^{n}(\theta)
\end{array}\right)=M(T)
$$

is a near-ring homomorphism of $N$ into a ring of $n \times n$ matrices over the

Presented to the Society, February 25, 1956; received by the editors July 12, 1955. 
real numbers. The kernel of this homomorphism is the two-sided ideal of $N$ consisting of all transformations in $N$ with vanishing total differential at $\theta$.

Let $T$ and $S$ be transformations in $N$ described by $F(X)$ and $G(X)$. The linearity of differentiation insures

$$
M(T+S)=M(T)+M(S) .
$$

That

$$
M(T S)=M(T) M(S)
$$

follows because the $j$ th partial derivative of $g^{i}(F(X))$ at $\theta$ is

$$
\sum_{k=1}^{n} g_{k}^{i}(\theta) f_{j}^{k}(\theta)
$$

Thus the correspondence $T \rightarrow M(T)$ is a near-ring homomorphism. The kernel of this homomorphism consists of those transformations in $N$ with vanishing total differential at the origin. Therefore these transformations form a two-sided ideal of $N$.

COROLlaRy 1. The ring of linear transformations of $V$ is a maximal simple near-ring in the near-ring of all transformations of $V$ which have $\theta$ as a fixed point and as a point of differentiability.

Let $N$ be a near-ring of transformations which have $\theta$ as a fixed point and as a point of differentiability. Suppose $N$ contains all the linear transformations and at least one nonlinear transformation $T$. If $L$ is the linear transformation with matrix $M(T), T-L$ is a nonlinear and, hence, nonzero transformation in $N$ with vanishing total differential at $\theta$. Theorem 1 now implies that $N$ has a proper twosided ideal and is not simple.

THEOREM 2. Let $N$ be a simple near-ring of transformations of $V$ which have $\theta$ as a fixed point, are differentiable at $\theta$, and include some transformation with nonvanishing total differential at $\theta$. If $X$ is a vector of $V$, the closure $\mathrm{Cl}(X N)$ of the set of vectors $X T$ for $T$ in $N$ is a subspace of $V$ invariant under $N$. If $\mathrm{Cl}(X N) \neq \theta$, the transformations in $N$ restricted to $\mathrm{Cl}(X N)$ faithfully represent $N$ as a simple subring of the ring of linear transformations of $\mathrm{Cl}(X N)$.

$N$ can be simple only if the homomorphism $T \rightarrow M(T)$ described in Theorem 1 is an isomorphism. Therefore $N$ is a ring. Because $N$ is a simple ring of characteristic zero, there is, for each transformation $T$ in $N$ and any integers $p$ and $q(\neq 0)$, a transformation $(p / q) T$ in 
$N$ such that

$$
X\left(\frac{p}{q} T\right)=\frac{p}{q}(X T)
$$

for all $X$ in $V$. Hence if $Y$ is in $\mathrm{Cl}(X N)$ and $\mu$ is a real number, $\mu Y$ is also in $\mathrm{Cl}(X N)$. That $\mathrm{Cl}(X N)$ is closed under addition follows because $X(T+S)=X T+X S$. This shows that $\mathrm{Cl}(X N)$ is a subspace of $V$.

Since $N$ is a ring

$$
(T+S) R=T R+S R
$$

for all $T, S, R$ in $N$. In particular

$$
(p T) R=(T+\cdots+T) R=T R+\cdots+T R=p(T R) .
$$

Hence

$$
p(X T R)=(p(X T)) R=\left(\frac{p q}{q}(X T)\right) R=q\left[\left(\frac{p}{q}(X T)\right) R\right]
$$

or

$$
\left(\frac{p}{q}(X T)\right) R=\frac{p}{q}(X T R)
$$

for all $X$ in $V$ and $T, R$ in $N$.

Suppose

$$
Y=\lim _{m \rightarrow \infty} X T_{m}
$$

For a transformation $R$ in $N$ select a nonzero rational number $p / q$ such that $R$ is continuous at $(p / q) Y$. This is possible because $R$ is differentiable and, hence, continuous in a neighborhood of $\theta$. Now

$$
\begin{aligned}
\frac{p}{q} \lim _{m \rightarrow \infty}\left(X T_{m} R\right) & =\lim _{m \rightarrow \infty}\left(\frac{p}{q} X T_{m} R\right)=\left(\lim _{m \rightarrow \infty} \frac{p}{q} X T_{m}\right) R \\
& =\left(\frac{p}{q} \lim _{m \rightarrow \infty} X T_{m}\right) R=\frac{p}{q} Y R
\end{aligned}
$$

and

$$
\lim _{m \rightarrow \infty} X T_{m} R=Y R
$$

Hence $R$ is continuous on $\mathrm{Cl}(X N)$. Since $X\left(T_{m} R\right)$ is in $\mathrm{Cl}(X N), Y R$ 
is in $\mathrm{Cl}(X N)$. It follows that $\mathrm{Cl}(X N)$ is an invariant subspace of $V$ with respect to $N$.

The continuity of $R$ on $\mathrm{Cl}(X N)$ together with (2) shows that

$$
(a Y) R=a(Y R)
$$

for $a$ real, $Y$ in $\mathrm{Cl}(X N)$, and $R$ in $N$. By (1)

$$
(X T+X S) R=X T R+X T S
$$

for $X$ in $V$ and $T, S, R$ in $N$. From the continuity of $R$ on $\mathrm{Cl}(X N)$

$$
(Y+Z) R=Y R+Z R
$$

for any $Y, Z$ in $\mathrm{Cl}(X N)$. By (3) and (4) the transformations in $N$ restricted to $\mathrm{Cl}(X N)$ are linear. Since the restriction of the transformations in $N$ to $\mathrm{Cl}(X N)$ effects a homomorphism of $N$, the simplicity of $N$ implies that this restriction gives a faithful representation of $N$ if $\mathrm{Cl}(X N) \neq \theta$.

The following example shows that the conclusion of linearity over $\mathrm{Cl}(X N)$ cannot be strengthened to linearity over $V$.

EXAMPLE 1. Let $V$ be two-dimensional and define the transformation $T_{a}$ for each real number $a$ by

$$
y_{1}=x_{1} f\left(x_{2}\right) a, \quad y_{2}=0
$$

where $f(x)$ is a fixed real function with $f(0)=1$ and $f^{\prime}(x)$ continuous at 0 . These transformations form a simple near-ring isomorphic to the field of real numbers. No $T_{a}(a \neq 0)$ is linear over $V$ unless $f(x)$ is constant.

ExAmple 2. Let $V$ be one-dimensional and define for each real number $a$ the transformation

$$
S(a): x \rightarrow y=|x| a .
$$

These transformations form a simple near-ring with the multiplication law

$$
S(a) S(b)=S(|a| b) .
$$

This example demonstrates the necessity of the differentiability assumption of Theorem 2.

ExAmple 3. Let $V$ and $S(a)$ be as in Example 2 and introduce the linear transformations

$$
T(a): x \rightarrow y=x a .
$$

The transformations of the form $T(a)+S(b)$ form a near-ring $N$ with the multiplication rules 


$$
[T(a)+S(b)] T(c)=T(a c)+S(b c)
$$

and

$$
\begin{aligned}
{[T(a)+S(b)] S(c) } & =S(|a| c)+T(|b| c) \quad \text { if } a b \geqq 0 \text { and }|a| \geqq|b|, \\
& =T(|a| c)+S(|b| c) \quad \text { if } a b \geqq 0 \text { and }|a| \leqq|b| \\
& =S(|a| c)-T(|b| c) \text { if } a b \leqq 0 \text { and }|a| \geqq|b|, \\
& =-T(|a| c)+S(|b| c) \text { if } a b \leqq 0 \text { and }|a| \leqq|b|
\end{aligned}
$$

These rules are derived from the simplification of

$$
|x a+| x|b|
$$

in the various cases.

Lemma 1. A nontrivial right module of $N$ consists of all elements $T(a r)+S(a)$ where $r$ is a fixed real number with $-1 \leqq r \leqq 1$ and a runs through all real numbers.

If

$$
T\left(a r_{1}\right)+S(a) \text { and } T\left(b r_{2}\right)+S(b) \quad(a b \neq 0)
$$

are elements of a right module $R$ of $N, R$ contains

$$
\left[T\left(a r_{1}\right)+S(a)\right] T(b)-\left[T\left(b r_{2}\right)+S(b)\right] T(a)=T\left(a b r_{1}-b a r_{2}\right) .
$$

If $r_{1} \neq r_{2}$ and $\Delta=a b r_{1}-b a r_{2}$,

$$
T(\Delta) T(c / \Delta)=T(c)
$$

is in $R$ for every c. Since $T\left(a r_{1}\right)+S(a)$ and $T\left(a r_{1}\right)$ are in $R, S(a)$ is in $R$. Hence

$$
S(a) T(d / a)=S(d)
$$

is in $R$ for every $d$. Therefore $T(c)+S(d)$ is in $R$ for all $c$ and $d$, and $R=N$.

If $T(a r)+S(a)$ is in $R$ and $|r|>1, R$ contains

$$
[T(a r)+S(a)] T(|a r|)=T(a r|a r|)+S(a|a r|)
$$

and

$$
\begin{aligned}
& {[T(a r)+S(a)] S(a)=S(|a r| a) \pm T(|a| a) } \\
&(+ \text { if } r \geqq 0 \text { and }- \text { if } r \leqq 0) .
\end{aligned}
$$

By subtraction

$$
T(a r|a r| \pm|a| a) \neq T(0)
$$


is in $R$. As above $R=N$.

If $T(a r)+S(a)(a \neq 0)$ is in $R, R$ contains

$$
[T(a r)+S(a)] T(b / a)=T(b r)+S(b)
$$

for all $b$. This combined with the previous facts shows that the only sets which could be nontrivial right modules of $N$ are those described in the lemma. Direct computation shows that these sets are right modules.

LEMMA 2. $N$ is a simple near-ring.

From Lemma 1 the intersection of two distinct nontrivial right modules of $N$ is $T(0)$. Therefore the right modules satisfy the descending chain condition. If $R$ is the right module of elements

$$
\begin{array}{ll}
\qquad T(a r)+S(a) & r \text { fixed with }|r| \leqq 1, \\
{[T(a r)+S(a)][T(b r)+S(b)]} & \\
=T\left(a b r^{2}\right)+S(a b r) \pm T(|a r| b)+S(|a| b) & (+ \text { if } r \geqq 0,- \text { if } r \leqq 0) \\
=T\left(a b r^{2} \pm|a r| b\right)+S(a b r+|a| b) . &
\end{array}
$$

If $b \neq 0, a b r-|a| b$ cannot be zero for both positive and negative values of $a$. Hence the product of nonzero elements of $R$ is not always zero, and $R$ is not nilpotent. If $b<0$,

$$
S(b)[T(a r)+S(a)]=S(b a r)+S(|b| a)
$$

is not in $R$. Therefore $R$ is not a left ideal. Thus there are no proper two-sided ideals, and $N$ is simple.

This example shows the necessity of the differentiability assumption in Theorem 2 and in Corollary 1.

EXAMPLE 4. Suppose $G$ is an additive subgroup of $V$ and $V^{0}$ is a subset of $V$ with $V^{0} \cap G=\theta$. Define the transformation $T_{g}$ for each $g$ in $G$ by

$$
\begin{array}{ll}
X T_{g}=g & \text { if } X \text { is not in } V^{0}, \\
X T_{g}=\theta & \text { if } X \text { is in } V^{0} .
\end{array}
$$

The transformations $T_{g}$ form a simple near-ring with the multiplication rules

$$
T_{g} T_{g^{\prime}}=T_{g^{\prime}} \quad(g \neq \theta)
$$

and

$$
T_{\theta} T_{g^{\prime}}=T_{\theta} .
$$

If $G$ is a discrete group $V^{0}$ may contain a neighborhood of $\theta$, so that 
each $T_{g}$ is differentiable at $\theta$ and has total differential zero at $\theta$. This shows the necessity of the hypothesis of Theorem 2 that there be at least one function in $N$ with nonvanishing total differential at $\theta$.

The $m$ th total differential at $A$ of a transformation $T$ described by $F(X)$ is the $n$-tuple

$$
\begin{aligned}
\Delta^{m} F(A)=( & \sum_{1}^{n} \cdots \sum_{1}^{n} f_{j_{1}}^{1} \cdots j_{m}(A) \Delta x_{j_{1}} \cdots \Delta x_{j_{m}}, \\
& \left.\cdots, \sum_{1}^{n} \cdots \sum_{1}^{n} f_{j_{1} \cdots j_{m}}^{n}(A) \Delta x_{j_{1}} \cdots \Delta x_{j_{m}}\right)
\end{aligned}
$$

where $f_{j 1}^{l} \cdots j_{m}(A)$ is the $m$ th partial derivative of $f^{i}(X)$ with respect to $x_{j_{1}}, \cdots, x_{j_{m}}$ evaluated at $A$. If all these partial derivatives exist in a neighborhood of $A$ and are continuous at $A$, then $T$ is $m$-times differentiable at $A$.

Theorem 3. Let $N$ be a near-ring of transformations of $V$ which have $\theta$ as a fixed point, are $m$-times differentiable at $\theta(m>1)$, have vanishing first $m-1$ total differentials at $\theta$, and include some transformation with nonvanishing mth total differential at $\theta$. Under these hypotheses $N$ is not simple.

The linearity of differentiation establishes that the transformations with vanishing $m$ th total differential at $\theta$ are an additive subgroup of $N$. That they form a two-sided ideal of $N$ follows because any product of two transformations in $N$ has vanishing $m$ th total differential at $\theta$. If this ideal is not the zero ideal, $N$ is not simple. If only the zero transformation has vanishing $m$ th total differential at $\theta$, every product of transformations in $N$ is zero so that $N$ is nilpotent and not simple.

Example 4 shows that the existence of a transformation with nonvanishing $m$ th total differential at $\theta$ is a necessary hypothesis in Theorem 3.

A transformation $T$ represented by $F(X)$ is analytic over $V$ if each coordinate function $f^{i}(X)$ can be expanded in a power series in $x_{1}, \cdots, x_{n}$ which converges throughout $V$.

COROLLARY 2. Every simple near-ring of analytic transformations of $V$ with $\theta$ as a fixed point is a simple ring which is faithfully represented as a ring of linear transformations by restricting the domain of the analytic transformations to the invariant subspace of $V$ generated by $a$ single nonzero vector not annihilated by $N$.

The analyticity of the transformations and Theorem 3 show that 
the simple near-ring contains a function with nonvanishing (first) total differential at $\theta$. The corollary follows by Theorem 2 .

COROLlaRY 3. The simple (nonzero) near-rings of entire functions over the complex plane which vanish at 0 are the rings of transformations described by equations

$$
w=a z
$$

where a ranges over a subfield of the complex numbers.

If the complex plane is regarded as a two-dimensional space $V$ over the real numbers, the entire functions are analytic transformations in the sense just defined. By Corollary 2 the transformations of the simple near-ring are linear on a (nonzero) subspace of $V$. From the complex analyticity of the functions, they are linear throughout $V$. Corollary 3 follows.

\section{REFERENCES}

1. D. W. Blackett, Simple and semisimple near-rings, Proc. Amer. Math. Soc. vol. 1 (1953) pp. 772-785.

2. W. E. Deskins, $A$ radical for near-rings, Proc. Amer. Math. Soc. vol. 5 (1954) pp. 825-827.

Boston UnIVERSITY 